\title{
Quantification of Japanese Quail Eggshell Colour by Image Analysis
}

\author{
METIN SEZER ${ }^{1}$ and OGUZ TEKELIOGLU ${ }^{2}$
}

Gaziosmanpasa University, Faculty of Agriculture, ${ }^{1}$ Department of Animal Science, ${ }^{2}$ Department of Agricultural Engineering, 60240, Tokat, Turkey

\begin{abstract}
The Japanese quail lays eggs with colourful and patterned shells which make the eggshell colour difficult to classify. In this study, the method of measuring colour of patchy eggs using image analyses and its power to discriminate among individual variation were established. Estimated repeatability for egg colour and proportion of patterned areas was high $(>0.58)$, suggesting intermediate or high heritability of eggshell colour characteristics. Three components have been identified as significant in discriminant function analysis. These three components explained $91.4 \%$ of the total variance in egg colour characteristics. In cluster analysis, $78.3 \%$ of the eggs that were collected from 15 females were correctly classified. This study indicates that eggshell colour characteristics can be reliably studied by image analyses and that this method can provide a unified character list for future examinations and interpretations of quail egg characteristics.
\end{abstract}

Key terms: eggshell colour, image analysis, individual variation, repeatability

\section{INTRODUCTION}

Remarkable variations in eggshell colour within and among avian species are common. Hence, shell colour of bird eggs is an interesting area for researchers from different disciplines, including avian biologists, evolutionary ecologists, and poultry breeders. Selection for crypsis, mimetism, filtering solar radiation, strengthening the eggshell, counter-defence against nest parasitism, and sexual selection as a signal of the genetic qualities of laying females have been suggested as the main evolutionary forces promoting the variation in avian egg colouration (Blanco and Bertellotti, 2002; Underwood et al., 2002; Moreno and Osorno, 2003; Gosler et al., 2005; Siefferman et al., 2005). Quantification of eggshell colour characteristics for concerned species is required to develop new hypotheses related to eggshell colour and to test existing ones. Accordingly, various categorical qualification systems for avian species have been preferred and applied in eggshell colour studies (Moreno and Osorno, 2003; Siefferman et al., 2005; Kirikci et al., 2005). Colour variation in spotless eggs is commonly determined using speciesspecific colour charts. This method is widely used to study eggshell colour variation within and among chicken breeds that lay brown-shelled eggs. Ranking by inexperienced observers is a preferred method in studies with spotted eggs. Lotem et al., (1995) successfully used this method to establish brood parasitism of the cuckoo, Cuculus canorus, on the great reed warbler, Acrocephalus arundinaceus. Visual classification was also employed to study the relationship between the quality and shell colouring of pheasant eggs (Kirikci et al., 2005).

The human visual system could be adequate to assess egg colour when the variation in egg pigmentation is high (Collias, 1993; Westmoreland and Kiltie, 
1996). However, image analysis has become an effective tool for evaluating biological data in a quick and reliable manner. Therefore, image analysis techniques in eggshell colour quantification have been used in various studies. Joseph (1998) was able to determine strain and diet effect shell colour in broiler breeder eggs using image analysis. Martens et al. (2005) presented the design of a computer vision system to differentiate and quantify the presence of different dirt stains on brown eggs. Evidence of eggshell colour as indicators of general condition and oxidative stress in female blue tits (Cyanistes caeruleus) has also been reported (Martinez-de la Puente et al., 2007).

Quail eggshell colour varies from white to blue and green. Additionally, quail eggs have brown or reddish-brown patterned areas on a light background (Figure 1). Unlike other plain pigmented avian eggs, colourful quail eggs, as a model system, provide more opportunities to study a wide variety of questions, such as the metabolism of pigment deposition, its relationship to overall bird physiology, egg quality and sexual behaviours. The available qualitative method to classify quail eggshell colour is based on the existence of spots on the eggshell surface. The eggs are graded as white, sandy-spotted, little-spotted, highspotted and/or medium-spotted (Okumus and Durmus, 1998). However, visual classification of eggshell colour is subjective and limits the statistical analysis that can be conducted. Hence, the aims of this study are to present the method of image analysis for quantifying quail eggshell colour and to test the proposed method's ability to distinguish variation among and within females.

\section{MATERIALS AND METHODS}

\section{Data collection}

The data for this study were obtained from the Japanese quail population (Cotunix coturnix japonica) in the Quail Breeding Unit of Gaziosmanpasa University, Tokat, Turkey. Female adult birds were housed in individual stainless steel wire mesh cages for accurate identification of eggs. Eggs

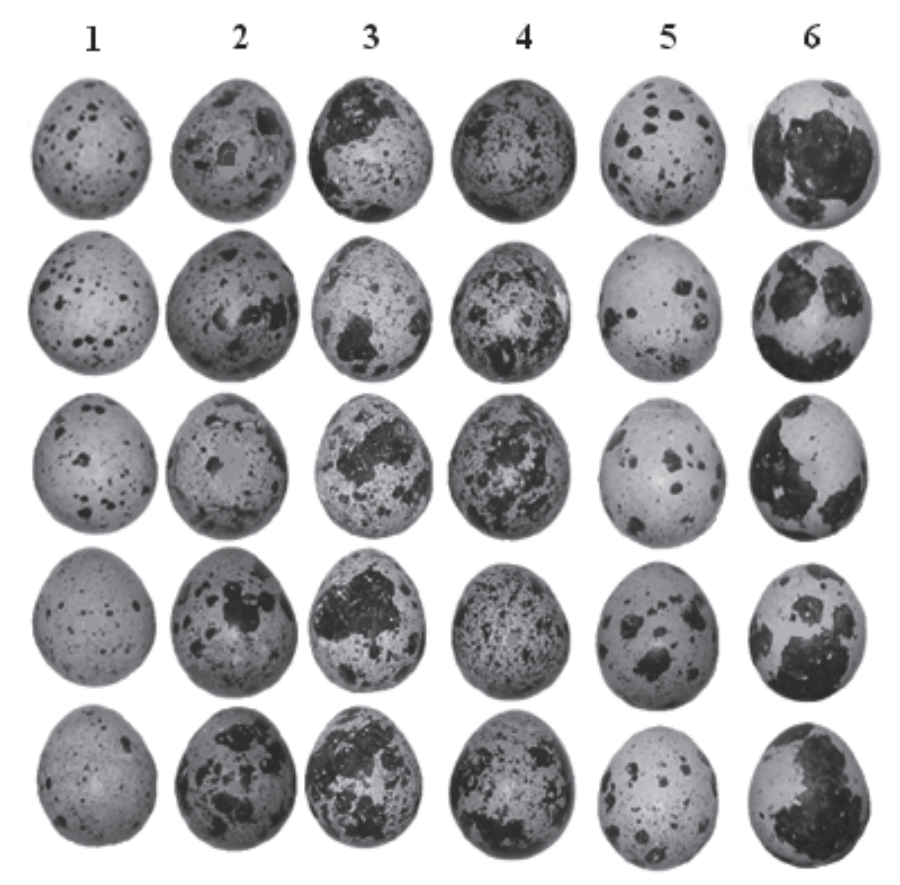

Figure 1: The range of variation of colour and patterns of eggshells within and among female Japanese quails (Coturnix coturnix japonica). 
were collected in 20 sequential days from 15 randomly chosen hens that were 12 weeks of age. Eggs with shells that were abnormal in shape, cracked, dirty or white were not used in the study. Considerable attention was given to the standardization of photographing conditions. Illumination was provided by a $4 \times 25 \mathrm{~W}$ lighting source surrounding the eggs. Images were taken 20 $\mathrm{cm}$ above the eggs using a digital camera (Canon Powershot A95) with 640 X 480 pixels of resolution and $256^{3}$ colours. Each egg was photographed from one side, and then turned $180^{\circ}$ to the other side and photographed again. The data obtained from both sides were averaged for the examined egg to reduce the error arising from pattern distribution over the eggshell. The total number of analysed images was 444, obtained from 222 eggs.

\section{Image analyses}

A Delphi-based program that is available at http://turhalmyo.gop.edu.tr/ogr/ ebu/ paket.htm was used to analyse the images. The region of interest (ROI), a rectangular area within the examined egg, was selected, the area being as large as possible. Because of the difference in the size and shape of the eggs, these areas were not constant. The program processes the ROI and calculates the mean values of intensity of the pixels in red, green and blue bands (Viscarra et al., 2006). These Red, Green and Blue (RGB) values were divided by 255 to produce a scale ranging from 0 to 1 . The formulas following Foley et al., (1996) were used to convert the RGB values into Hue, Saturation and Lightness (HSL) values.

Categorical classification of the Japanese quail eggshell colour was based on the distribution of markings over a light background. Hence, the proportions of the light background to ROI were also calculated (Figure 2). The image analysis program can search the entire image and select the pixels having similar colour band intensities as a desired pixel. Then, selected pixels can be converted to a distinct colour that is not represented in the eggshell (e.g. pure red: $255,0,0: R, G, B)$. Using this specification of the program, the total number of pixels in the ROI and converted pixels for the patterned areas were counted. Finally, the proportions of the pixels in the patterned area to the total number of pixels in the ROI were calculated.

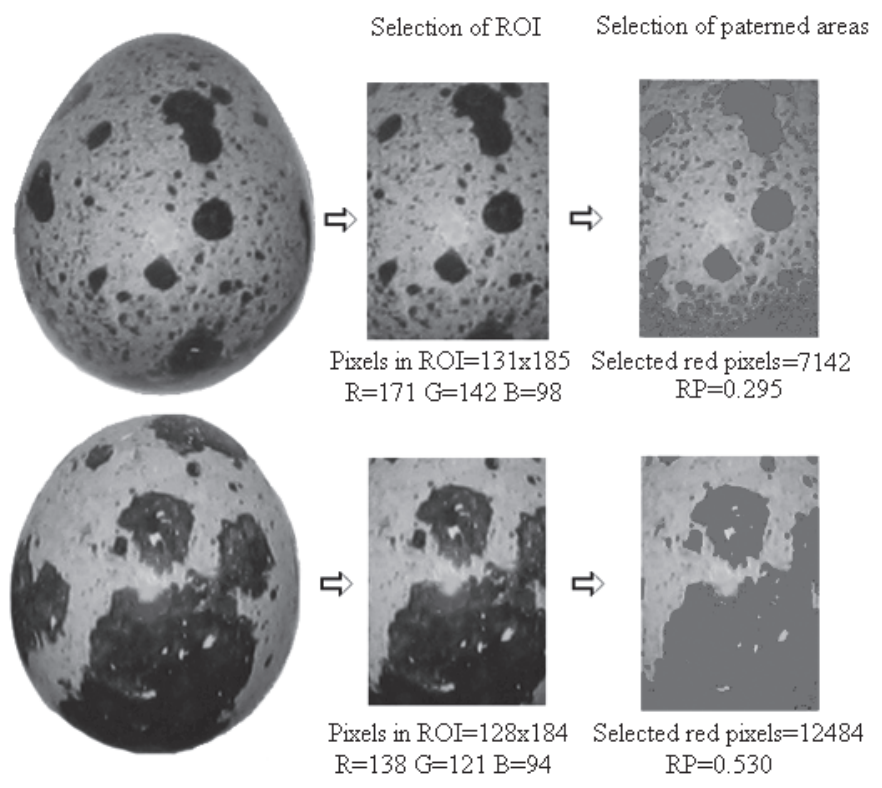

Figure 2: Selection of the region of interest (ROI) and calculation of the proportions of the patterned areas to the ROI (RP). 


\section{Statistical analyses}

Colour characteristic values of the eggs were subjected to discriminant function analysis (DA) to determine which variables discriminate eggshell colour and to identify which eggs belong to a specific hen based on observed characteristics of each egg. DA reduces a set of $p$ variables to $m$ components or factors prior to further analyses on those $m$ factors. Each discriminant function is that which maximally separates the groups and filters redundant information. The power of this analysis is affected by the number and novelty of the variables. Because of the high correlations among the Red, Green and Blue values (Foley et al., 1996), data obtained from RGB and HSL colour space values were jointly used in the discriminant function analysis.

Examination of variation both among and within individuals is required to understand the evolution of traits. Repeatability is defined as the ratio of trait variation among individuals to the sum of trait variation among and within individuals (Boake 1989; Falconer and Mackay 1996). Higher variation among individuals compared to that within individuals, i.e. high repeatability, suggests the possibility of the existence of high heritability values since repeatability sets the upper bound of heritability (Boake 1989). The repeatability of egg colour measurements and standard errors of repeatability were calculated using a one-way analysis of variance procedure following Lessells and Boag (1987) and Becker (1992). SPSS 10.0 for Windows was used for all of the statistical analyses.

\section{RESULTS}

Shell colour and pattern of Japanese quail eggs are highly consistent within females and considerable variation is visible among individuals (Figure 1). Eggshell colour was significantly discriminated in the percentage of patterned areas, RGB and HSL spectra $(\mathrm{P}<0.01)$. Repeatability is a measure of the proportion of variance in a character that occurs among, rather than within individuals (Lessells and Boag, 1987; Falconer and Mackay, 1996). Hence, measures of repeatability could be used to assess patterns of variation in egg characteristics within and among females. Mean values and repeatability calculated for egg colour characteristics and proportion of patterned areas were presented in Table 1. Repeatability for egg colour and proportion of patterned areas were high $(>0.58)$.

\section{TABLE 1}

Mean over the females $( \pm$ standard deviation), and repeatability ( $\mathrm{r} \pm$ standard error) of the examined colour characteristics

\begin{tabular}{lcc}
\hline Traits & Mean \pm SD & $\mathrm{r} \pm \mathrm{SE}$ \\
\hline RP $^{*}$ & $0.63 \pm 0.156$ & $0.85 \pm 0.051$ \\
Red & $0.63 \pm 0.084$ & $0.60 \pm 0.097$ \\
Green & $0.57 \pm 0.092$ & $0.70 \pm 0.085$ \\
Blue & $0.43 \pm 0.091$ & $0.79 \pm 0.067$ \\
Hue & $42.10 \pm 5.35$ & $0.76 \pm 0.073$ \\
Saturation & $0.24 \pm 0.063$ & $0.58 \pm 0.099$ \\
Lightness & $0.53 \pm 0.084$ & $0.71 \pm 0.083$ \\
\hline
\end{tabular}

${ }^{*} \mathrm{RP}=$ ratio of the patterned areas to the region of interest

Shell colour and pattern characteristic values of eggs were subjected to discriminant function analysis to determine the variables describing eggshell colour of the Japanese quail and to establish the identification accuracy of the eggs obtained from known females. Three components have been identified as significant (eigenvalue $>1$ ). These three components explained $91.4 \%$ of the total variation in egg colour characteristics. The structure matrix for these components is presented in Table 2.

The first component, which included the variables for the blue spectra and the proportion of patterned areas to the ROI, described $46 \%$ of the variation between quail eggs. The second component, which included the proportion of patterned areas and saturation variable, described an additional $33.9 \%$ of the variation. The third 
component, which included the Hue variable, described an additional $11.4 \%$ of the variation. Discriminant scores for each egg from the first and the second function were used to calculate the means and standard deviations for females and are presented in Figure 3. A majority of the eggs obtained from the 15 females can easily be discriminated from the others using only the first two discriminant scores.

TABLE 2

Structure matrix for the component obtained from discriminant function analysis.

\begin{tabular}{lccc}
\hline Traits & \multicolumn{3}{c}{ Component } \\
\cline { 2 - 4 } & 1 & 2 & 3 \\
\hline RP* & 0.722 & 0.635 & -0.213 \\
Blue & 0.678 & -0.119 & 0.517 \\
Hue & 0.520 & -0.341 & -0.588 \\
Saturation & -0.112 & 0.478 & -0.055 \\
Red & 0.413 & 0.183 & 0.263 \\
Green & 0.563 & 0.044 & 0.227 \\
Lightness & 0.569 & 0.051 & 0.395 \\
\hline
\end{tabular}

${ }^{*} \mathrm{RP}=$ ratio of the patterned areas to the region of interest.
When egg colour values were used in cluster analysis, $78.3 \%$ of the eggs were correctly classified. Eggs of 10 females were correctly classified with accuracy greater than $75 \%$. The lowest correct classification value for a female using the colour characteristics of the eggshell was $53.8 \%$.

\section{DISCUSSION}

Egg predation may favour crypsis in eggshell colour, resulting in the eggshell resembling the nest and nesting place. Therefore, egg coloration is highly variable among species because of the great variety of materials observed in nests and nesting sites (Westmoreland \& Kiltie, 1996; Blanco and Bertellotti, 2002). Quails are ground nesting birds and their multicoloured eggshells give this necessary advantage to protect their eggs from predators.

Significant differences in eggshell colour characteristics among females indicate that quantification of eggshell colour using image analysis could be used to assess eggshell colour and pattern. The results of the discriminant function analysis

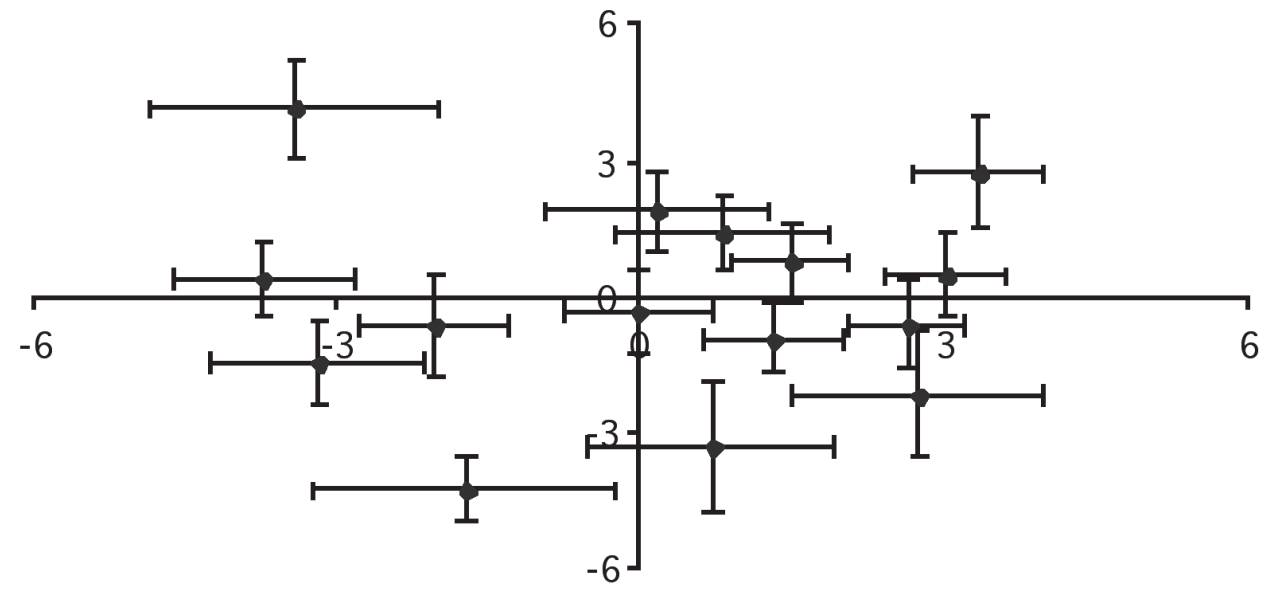

Component 1

Figure 3: Means and standard deviations of discriminant scores for the first and the second function for each female. 
demonstrate that the patterns on eggshells are an important variable to describe eggshell pigmentation. Carotenoid pigments are known to have antioxidant properties. They scavenge harmful free radicals and protect cells, tissues and the immune system from oxidative injury (Krinsky 2001, McGraw, 2005). Hence, Moreno and Osorno (2003) have discussed the possibility that the eggshell colour of avian species with biparental care might function as a signal of female phenotypic or genetic qualities to their mates, like other colourful sexual ornaments. Siefferman et al. (2006) have found a positive correlation between eggshell colour and female condition in the Eastern Bluebird, Sialia sialis. Similarly, evidence of eggshell colour as an indicator of general condition and oxidative stress in the female Blue Tit (Cyanistes caeruleus) has been reported (Martinez-de la Puente et al., 2007). It has also been reported that intensity of blue-green coloration was significantly related to the duration of the nesting period and to the degree of polygyny in passerines (Soler et al., 2005). Alternatively, there may be health related reasons to use pigments in eggshell. Experimental evidence for a correlation between the female nutritional condition and eggshell colour was provided by Moreno et al. (2006). They have found that blue-green egg colour reflects the maternal antibody content and nestling survival probability in the song bird, Ficedula hypoleuca. Identification of Japanese quail eggs using colour characteristics could very possibly lead to better identification of female variability and provide a unified character list for future biological examinations and interpretations.

Although eggshell colour variation among Japanese quails was high, variation within individual females was considerably low. Low variation within females has been suggested to be utilized as an individual egg recognition system in dense nestingsites, and a protection against nest parasitism (Blanco and Bertellotti, 2002; Soler et al., 2005). Similar to our study, cluster analysis was performed with a combination of variables for volume, surface area and shapes to compare the eggs of Emperor Geese (Petersen, 1992). Petersen was able to discriminate among the eggs within clutches with an accuracy of $77.8 \%$. Individual variations in egg colour and pattern were also reported for other avian species (Collias, 1993; Westmoreland and Kiltie, 1996; Kim et al., 1996). Estimated repeatability of egg characteristics for the Japanese, quail such as mass, volume, length, and shape index was higher than that estimated for eggshell characteristics of other avian species, (Christians, 2002). Higher variation among individuals compared to within individuals suggests that eggshell colour characteristics might be useful for determining the individual identity of female Japanese quails. This pattern of variation could be a result of directional selection for efficient brood and parasitic egg recognition. Repeatability comprises heritability and general environmental variance. However, general environmental variance is expected to be low in this data set. These results also suggest that intermediate or high heritability of eggshell colour characteristics might be expected.

The colour chart and other categorical quantification methods are designed to reflect human perception of colour and its variations. Hence, the principle reason with respect to the lack of detailed studies, especially on species with multi-coloured eggs, was unavailability of a quantitative method of measurement of shell colour. In this study, the method of measuring colour of patchy eggs and its ability to discriminate among individual variation were established. Our results provide valuable clues towards further analyses of the physiological and genetic bases of eggshell colour variation that may perhaps elucidate the causes of intraspecific variation.

\section{REFERENCES}

1. BECKER WA (1992) Manual of Quantitative Genetics. Pullman: WA Academic Enterprises

2. BLANCO G, BERTELLOTTI M (2002) Differential predation by mammals and birds: Implications for eggcolour polymorphism in a nomadic breeding seabird. Biol J Linn Soc 75: 137-146 
3. BOAKE CRB (1989) Repeatability: its role in evolutionary studies of mating behavior. Evol Ecol 3: 173-182

4. CHRISTIANS JK (2002) Avian egg size variation within species and inflexibility within individuals. Biol Rev 77: $1-26$

5. COLLIAS EC (1993) Inheritance of egg-color polymorphism in the village weaver (Ploceus cucullatus). The Auk 110: 683-692

6. FALCONER DS, MACKAY TFC (1996) Introduction to Quantitative Genetics. New York: Longman

7. FOLEY JD, VANDAM A, FEINER SK, HUGHES JF (1996) Computer Graphics Principles and Practice. New York: C. Addison-Wesley Press

8. GOSLER AG, HIGHAM JP, REYNOLDS SJ (2005) Why are birds eggs speckled?, Ecol Let 8: 1105-1113

9. JOSEPH N (1998) Can eggshell quality be determined by shell color? http: //www.thepoultrysite.com/articles/ 576/can-eggshell-quality-be-determined-by-shell-color. Accessed March 2007

10. KIM CH, YAMAGISHI S, WON PO, (1995) Egg-color dimorphism and breeding success in the crow tit (Paradoxornis webbiana). The Auk. 112: 831-839

11. K1RIKCI K, GUNLU A, GARIP M (2005) Some quality characteristics of pheasant (Phasianus colchicus) eggs with different shell colors. Turk J Vet Anim Sci 29: 315-318

12. KRINSKY NI (2001) Carotenoids as antioxidants. Nutrition 17: 815-817

13. LESSELLS CM, BOAG PT (1987) Unrepeatable repeatabilities A common mistake. The Auk 104: 116121

14. LOTEM A, NAKAMURA H, ZAHAVI A (1995) Constraints on egg discrimination and cuckoo-host coevolution. Anim Behav 49: 1185-1209

15. MARTÍNEZ-DE LA PUENTE J, MERINO $S$, MORENO J, TOMA'S G, MORALES J, LOBATO E, GARCÍA-FRAILE S, MARTÍNEZ J (2007) Are eggshell spottiness and colour indicators of health and condition in blue tits Cyanistes caeruleus? J. Avian Biol. 38: 377-384

16. MCGRAW KJ (2005) The antioxidant function of many animal pigments: are there consistent health benefits of sexually selected colourants? Animal Behaviour 69: 757-764

17. MERTENS K, DEKETELAERE B, KAMERS B, BAMELIS FR, KEMPS BJ, VERHOELST EM, DEBAERDEMAEKER JG, DECUYPERE EM (2005) Dirt detection on brown eggs by means of color computer vision. Poult Sci 84: 1653-1659

18. MORENO J, LOBATO E, MORALES J, MERINO S, TOMA'S G, PUENTE JM, SANZ JJ, MATEO R, SOLERC JJ (2006) Experimental evidence that egg color indicates female condition at laying in a songbird. Behav Ecol 17: 651-655

19. MORENO J, OSORNO JL (2003) Avian egg colour and sexual selection Does eggshell pigmentation reflect female condition and genetic quality?. Ecol Let 6: 803806

20. OKUMUS A, DURMUS I (1998) Egg color genes and its quantitative utilisation in Japanese quail. Pages 2126 in $10^{\text {th }}$ European Poultry Conf., Jerusalem, Israel

21. PETERSEN MR (1992) Intraspecific variation in egg shape among individual Emperor Geese. J Field Ornithol 63: 344-354

22. SEIFFERMAN L, NAVARA KJ, HILL GE (2006) Egg coloration is correlated with female condition in eastern bluebirds (Sialia sialis). Behav Ecol Sociobiol 59: 651-656

23. SOLER JJ, MORENO J, AVILES JM, MØLLER AP (2005) Blue and green egg-color intensity is associated with parental effort and mating system in passerines support for the sexual selection hypothesis. Evolution 59: 636-644

24. UNDERWOOD TJ, SEALY SG (2002) Adaptive significance of egg coloration. In: Deeming DC (Ed). Avian Incubation, Behaviour, Environment and Evolution. Oxford University Press. pp: 280-298

25. VISCARRA ROSSEL RA, MINASNY B, ROUDIER P, MCBRATNEY AB (2006) Colour space models for soil science. Geoderma 133: 320-337

26. WESTMORELAND D, KILTIE RA (1996) Egg crypsis and clutch survival in three species of blackbirds (Icteridae), Biol J Linn Soc 58: 159-172 